\title{
A Comparative Evaluation of Touch and Pen Gestures for Adult and Child Users
}

\author{
Ahmed Sabbir Arif \\ York University \\ Toronto, Ontario, Canada \\ a.s.arif@gmail.com
}

\author{
Cristina Sylla \\ engageLab, University of Minho \\ Guimarães, Portugal \\ sylla@engagelab.org
}

\begin{abstract}
In this paper, we present results of two user studies that compared the performance of touch-based and pen-based gesture input on capacitive touchscreens for both adult and 8-11 years old child users. Results showed that inputting gestures with pen was significantly faster and more accurate than touch for adult users. However, no significant effect of input method was observed on performance for child users. Similarly, user experience evaluation showed that a large number of adult users favoured one technique over the other and/or found a technique more comfortable to use than the other, while child users were mostly neutral. This trend, however, was not statistically significant.
\end{abstract}

\section{Categories and Subject Descriptors}

H.1.2 [Information Systems]: User/Machine Systems - Human factors.

\section{General Terms}

Human Factors, Measurement, Performance.

\section{Keywords}

Children, gesture input, mobile, pen, text entry, touch.

\section{INTRODUCTION}

Touchscreens have become a dominant modality for handheld devices. Touchscreen-based devices are in demand and current trend promises more in the near future. Most of these devices use capacitive touchscreens where finger-input is the only interaction method. This is mainly because: 1) users are not required to constantly carry "easy to lose" styli to interact with such devices, and 2) touch interaction is generally assumed to be a more natural and fluid method of interaction than pen. This, however, is a substantial change from the earlier trend where styli were the dominant input method for mobile phones and tablets [13]. This demands empirical investigation on how quantitatively different finger-based and pen-based gestures are, in terms of speed and accuracy. Unfortunately, not much work has been done regarding this. Most research $[1,6,15]$ examined the performance of high quality inductive digital styli that do not apply to finger gestures. Nevertheless, in a recent work [14] finger and pen gestures with different degrees of complexity and target sizes were compared. Results showed that drawing complex gestures with pen takes less

Permission to make digital or hard copies of all or part of this work for personal or classroom use is granted without fee provided that copies are not made or distributed for profit or commercial advantage and that copies bear this notice and the full citation on the first page. Copyrights for components of this work owned by others than ACM must be honored. Abstracting with credit is permitted. To copy otherwise, or republish, to post on servers or to redistribute to lists, requires prior specific permission and/or a fee. Request permissions from Permissions@acm.org.

IDC '13, June 24 - 27 2013, New York, NY, USA

Copyright 2013 ACM 978-1-4503-1918-8/13/06 \$15.00. time than touch.

Touchscreen-based devices are becoming popular amongst child users as well [8]. Numerous touch-based devices and applications are available in the market for children and more are being developed. It is well known that children are a special user-group that requires certain considerations, as they have different needs, desires, and expectations than adult users. Therefore, results of the studies conducted with adult users are not applicable to them. Although, recently there has been a growing interest in exploring how children interact with touchscreens, most of this work focused on either children's capability of using touch or pen $[4,9$, $10]$ or designing user interfaces for children [2, 12]. To our knowledge no research has been done to examine the quantitative difference between finger and pen gestures for child users.

Here, we present results of two user studies that compared touch and pen gestures for both adult and 8-11 years old child users. Results showed that inputting gestures with pen was significantly faster and more accurate than touch for adult users. However, no significant effect of method was found for child users. Both user groups were comfortable inputting gestures with both touch and pen. They were also mostly positive regarding using either of these techniques dominantly on touchscreen-based devices.

\section{MOTIVATION}

Although, touch has become the dominant method for interacting on mobile devices, a number of recent devices still support penbased interaction. Besides, many third-party capacitive pens are available for touch-only devices. Digital pens or styli are usually used for tasks such as drawing, sketching, or note taking.

Currently, a substantial number of child users use mobile devices as well [8]. Interestingly, almost all recent mobile applications for child users are touch-based. It is, however, almost impossible for designers to make an informed decision on which method to pick (i.e. touch or pen) as currently there is not enough data on how different these two methods are in terms of performance and user acceptance. Thus, to initiate research interest in this direction, we investigated the difference between touch and pen gesture input, not only for adult users but also for 8-11 years old child users.

\section{USER STUDIES}

First, we discuss the apparatus, procedure, and performance metrics that were used for both studies.

\subsection{Apparatus}

A first generation Apple iPad Wi-Fi with 9.7" multitouch display at $1024 \times 768$ pixels was used during the study with the adult users. A third generation Apple iPad Wi-Fi with 9.7" multitouch display at $2048 \times 1536$ pixels was used with the child users. Two different iPads were used as the two studies were conducted in different labs. We, however, do not believe this to have an effect on the study results. Commercial iPad cases were used to tilt the devices 
into a comfortable $\sim 11^{\circ}$ typing position, see Figure 3 . A custom Web application was developed with HTML5 and JavaScript and used during the user studies. It was launched on the same version of the default iPad Safari browser during the studies, see Figure 1. Once cached, the application did not rely on the Internet to obtain data. This eliminated the risk of system delay or lag due to connectivity. The application used the $\$ 1$ recognizer [15] to process gestures. It logged all interactions with timestamps and calculated user performance directly. At the end of each session the application buffered all data with a database using the devices' wireless connection.

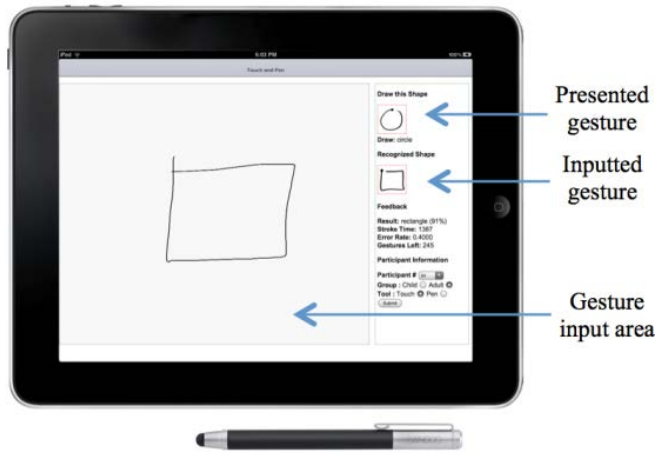

Figure 1. The application used during the studies. Below is the Bamboo Stylus. The Apple iPad and the pen are not to scale.

We used a Wacom Bamboo Stylus for iPad to allow pen-based input, weight 20 gram, length $120.8 \mathrm{~mm}$, diameter $9.0 \mathrm{~mm}$, and tip-diameter $6 \mathrm{~mm}$. See Figure 1. This pen was selected based on a study [11] that showed that the most suitable pen for adult users is the one with length $110-150 \mathrm{~mm}$, width $7 \mathrm{~mm}$, and tip-width 1 $1.5 \mathrm{~mm}$. Then, for $10-11$ years old child users, length $70-130 \mathrm{~mm}$, width $4 \mathrm{~mm}$, and tip width 1-1.5 mm. The Wacom Bamboo Stylus satisfied both age groups respecting length and width. Although, the pen-tip was substantially thicker, it provided one of the closest to the optimal pen-tip thickness amongst the ones available in the market. Also, its resemblance to a real pen made it easier for child users to get accustomed to it.

\subsection{Procedure}

Nine unistroke gestures were selected for the studies. See Figure 2. These gestures were used with both the adult and the child users to provide a better comparison between the groups. The gestures were selected using the CLC model [3] that computes a gesture's production time based on sub-models of curve, line, and corner production; using criteria similar to Tu et al.'s [14]. Only "simple" gestures were selected considering child users.

We decided to use unistroke gestures as multistroke often permit different methods for drawing the same shape. This makes it harder to compare gestures that are alike but drawn in different ways. Also, we consulted three experienced preschool teachers regarding this. All of them stated that unistroke gestures are easier for 8-11 years old children to draw.

The application presented one gesture at a time on the screen. Participants then had to input the presented gesture using either touch or pen. There were two blocks: one for touch and the other for pen input. Users were instructed to input the gestures as fast and accurate as possible. They were provided with visual feedback on drawing. That is, users could see what they had drawn so far in real time. Timing (and a stroke) started from the moment the users started drawing a shape and ended when they lifted their finger or the pen. The next gesture was displayed on the screen when the users finished inputting the presented gesture. The last drawn shape remained on the canvas until they started drawing the newly presented shape. Error correction was not forced. That is, users were not required to correct their mistakes when an incorrect gesture was drawn or the system misrecognized it. The intention was to exclude correction efforts from the calculation of the error rate and average stoke time. However, users were provided with auditory and visual feedback on erroneous input or misrecognized gestures. The system played a "ding" sound and displayed a special symbol on the screen. See Figure 2. This aided users to identify probable erroneous actions and to correct those in later attempts.

Before each block, users inputted 20 practice gestures using the corresponding method. They could extend the duration of their practice, if felt necessary. Users were instructed that they could rest between blocks and/or before inputting the gestures. The capacitive touchscreen was wiped clean using a dry cloth after each block. Upon completion of the study, adult users were asked to fill out a short questionnaire where they could rate the input methods on a five-point Likert scale and comment on them. The examiner interviewed the child users, in contrast, to make sure that they understood the questions. Each session took about 30 minutes, for both adult and child users, including demonstration, practice blocks, and breaks.

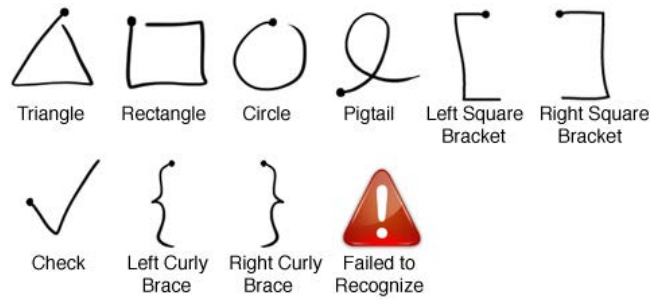

Figure 2. The nine gestures used during the studies. Here, a dot denotes the start of a stroke. The last symbol was used to provide visual feedback on incorrect attempts.

\subsubsection{Performance Metrics}

We calculated the following measures during the user studies:

- Stroke Time (milliseconds): This denotes on average how much time it took to draw one gesture.

- Error Rate (\%): This denotes on average how many gestures were drawn incorrectly or misrecognized by the system. This, however, does not include error correction efforts.

\subsubsection{User Feedback}

Users provided feedback on the following factors:

- Physical Comfort: The overall physical comfort of using an input method on touchscreens.

- Willingness to Use: Users' interest in using an input method dominantly on touchscreen-based devices to draw gestures.

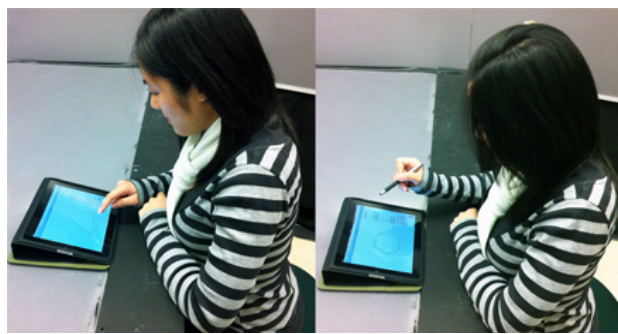

Figure 3. An adult participant inputting gestures using touch (left) and pen (right). 


\section{USER STUDY 1: ADULT USERS}

\subsection{Participants}

Twelve participants, aged from 21 to 30 years, average 26, took part in the study. Six of them were female and all of them were right-handed. Eight users had experience with touchscreen-based devices and three had experience with pen-based devices. They all received a small compensation, that is, CAD $\$ 5.00$ for participating in the study.

\subsection{Design}

We used a within-subject design. There were two blocks: touch and pen. In each block, users entered 250 gestures with a different method (500 gestures, in total). They were randomly assigned into groups with interchanged blocks based on a Latin Square in order to avoid asymmetric skill transfer. In summary, the design was:

12 participants $\times$

2 blocks (touch and pen) $\times$

250 gestures

$=6000$ gestures in total, excluding practice gestures.

\section{USER STUDY 2: CHILD USERS}

\subsection{Participants}

Twelve children, aged from 8 to 11 years, average 9, participated in this study. The age-range was decided based on prior research $[5,7]$ that showed that children under 7 have difficulties to draw shapes. Therefore, all of our participants knew the ergonomic way to hold a pen and used the tripod grasp during the study. Six participants were male and all were right-handed. Eight of them had experience with touch-based devices, but none had experience with pen-based devices. All children received a chocolate bar for participating in the study.

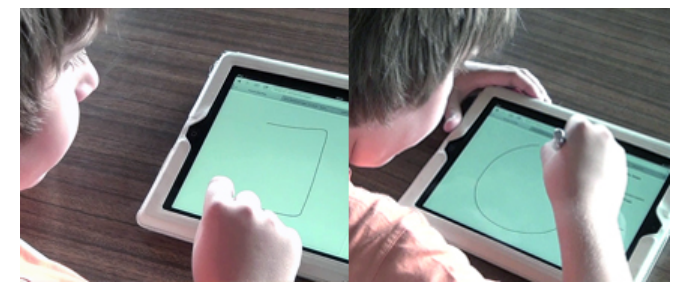

Figure 4. A child participant inputting gestures using touch (left) and pen (right).

\subsection{Design}

The design was similar to the first study but with one exception. That is, in each block child users inputted 150 gestures instead of 250 , in total 300 . This number was picked based on a pilot study where we observed that child users often suffer from physical or mental discomfort when asked to input more than 150 gestures. In summary, the design was:

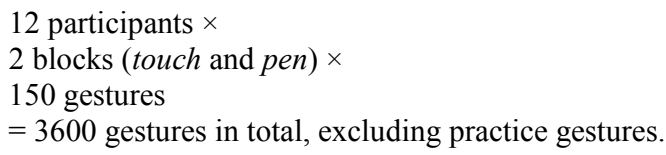

\section{RESULTS}

An Anderson-Darling Test on the study data revealed that the data was normally distributed. Therefore, we used repeated-measures ANOVA for all intragroup analyses. However, Kruskal-Wallis one-way ANOVA was used for all other analyses.

\subsection{Stroke Time}

A significant effect of age group on stroke time was identified for both touch $\left(H_{1}=970.41, p<.0001, \eta^{2}=.17\right)$ and pen $\left(H_{1}=1120.55\right.$, $\left.p<.0001, \eta^{2}=.19\right)$. For adult users, the effect of input method on stroke time was significant $\left(F_{1,11}=8.46, p<.05, \eta^{2}=.01\right)$. Drawing gestures with pen was significantly faster than touch. For child users, however, the effect of input method on entry speed was not significant $\left(F_{1,11}=8.46, \mathrm{~ns}\right)$. Figure 5 illustrates the average stroke time for adult and child users with both touch and pen.

\subsection{Error Rate}

A significant effect of age group on accuracy was identified for both touch $\left(H_{1}=80.60, p<.0001, \eta^{2}=.02\right)$ and pen $\left(H_{1}=120.30\right.$, $\left.p<.0001, \eta^{2}=.02\right)$. For adult users, the effect of input method on accuracy was significant $\left(F_{1,11}=5.94, p<.05, \eta^{2}=.002\right)$. Drawing gestures with pen was significantly more accurate than touch. However, similar to entry speed, there was no significant effect of input method on accuracy $\left(F_{1,11}=0.31, \mathrm{~ns}\right)$ for child users. Figure 6 illustrates average error rate for touch and pen for both groups.

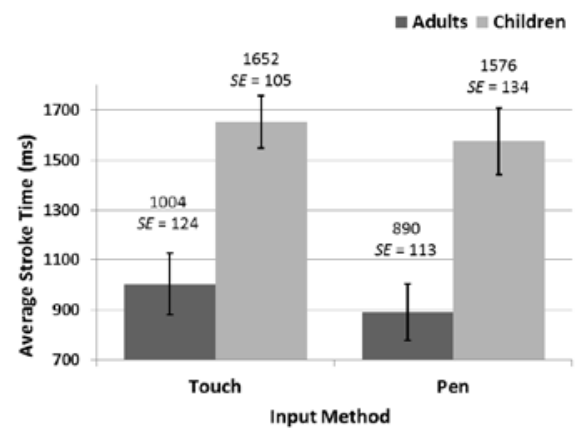

Figure 5. Average stroke time (milliseconds), with standard error (SE), while drawing with touch and pen, for both age groups. Note the vertical scale.

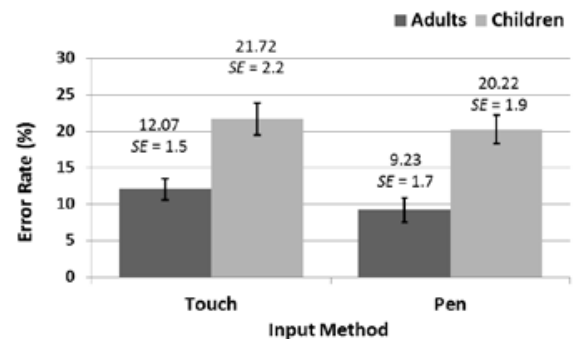

Figure 6. Average error rate (\%), with standard error (SE), while drawing with touch and pen, for both age groups.

\subsection{Physical Comfort}

Analysis on the feedback data did not identify a significant effect of input method on the overall comfort for adult users $\left(H_{1}=0.42\right.$, ns). $25 \%$ found touch, $33.33 \%$ found pen, $33.33 \%$ found both, and $8.34 \%$ found none of the methods comfortable to use. There was also no significant effect for child users $\left(H_{1}=3.14\right.$, ns). $8.33 \%$ found touch, $25 \%$ found pen, and $66.67 \%$ found both methods comfortable to use.

\subsection{Willingness to Use}

Analysis on the feedback data indicated that neither adult $\left(H_{1}=1.51, \mathrm{~ns}\right)$ nor child users $\left(H_{1}=1.05, \mathrm{~ns}\right)$ favoured one input method significantly over the other. Amongst adult users, $66.67 \%$ preferred touch, $16.67 \%$ preferred pen, and the rest $16.66 \%$ were neutral. Amongst child users, $16.67 \%$ preferred touch, $16.67 \%$ preferred pen, and $66.66 \%$ were neutral.

\section{DISCUSSION}

Pen yielded a significantly better performance in terms of speed and accuracy for adult users. However, the overall performance for touch and pen were relatively similar for child users. This 
indicates towards the possibility that pen becomes a more effective input method over time with practice as one starts writing with pens (on papers) dominantly. The fact that our results did not show a significant effect of input method on speed and accuracy for child users is also interesting as a prior work [8] reported that child users usually dislike pen gestures. Hence, our results must be encouraging to interface designers for children. Note that the error rate was very high for child users with both touch and pen. During the study, we noticed that a large number of these errors were committed through accidental touches on the screen. Sometimes children would lift their finger or pen in the process of drawing a shape, usually because they did not like the shape they just drew and wanted to re-draw it. This behaviour is not unusual, as most children in school are allowed to redesign a shape if they do not like it. No significant effect was found in terms of physical comfort for both adult and child users. A large number of adult users $(57.33 \%)$, however, found one technique to be more comfortable than the other, while most child users $(66.67 \%)$ were neutral. Similar trend was observed regarding willingness to use. A large number of adult users $(83.34 \%)$ preferred one technique to the other, while most child users $(66.66 \%)$ were neutral. This indicates towards the possibility that mobile users start favouring an interaction technique as they become accustomed to it. This favouritism may not be always dependent on performance, as during our study most adult users $(66.67 \%)$ preferred touch, although they evidently performed better with pen. Further investigation is required in this direction.

\subsection{Adult vs. Child Users}

This article should encourage researchers to further investigate touch and pen gesture input for child users. Results showed that child users are almost equally comfortable with touch and pen and do not prefer one technique to the other. There was also no significant difference in performance. This suggests that either or both of these techniques could be used on systems for child users.

Adult users displayed a different and at the same time interesting trend. They performed significantly better with pen, but preferred using touch. We speculate: they performed better with pen as they all have extensive experience with it, as they all started using pens at a very early stage. They preferred touch, because almost all of them owned a touch-based device and got accustomed to it. Further investigation is required to fully support our hypotheses.

\section{CONCLUSION AND FUTURE WORK}

Here, we presented results of two user studies that compared the performance of touch and pen gesture input for adult and child users. Results showed that adults were significantly faster and more accurate while inputting gestures with a pen than with touch. However, no significant effect of input method on performance was found for children. Although statistically insignificant, user experience evaluation showed that a large number of adult users favoured one technique over the other and/or found a technique more comfortable to use than the other, while child users were mostly neutral.

In the future, we intend to conduct a series of user studies to better understand how children interact with touchscreens. The intention would be to provide designers and practitioners with guidelines, to assist them with designing efficient user interfaces for children. Also, this work did not take gesture size into consideration. Thus, we would like to investigate whether scale has a significant effect on performance or not. Moreover, during the studies, users were not allowed to rest their palm on the touch surface. In the future, we would like to use a palm-rejection technique to provide the users with a more fluid and natural interaction. Finally, we would like to examine touch and pen gesture input on both capacitive and resistive screens, with different types of pens or styli.

\section{ACKNOWLEDGMENTS}

Part of this work was funded by FEDER through the Operational Competitiveness Factors Program - COMPETE and by National Funds through the FCT - Portuguese Foundation for the Science and the Technology within the Project: PTDC/CPE-CED/110417 12009 .

\section{REFERENCES}

[1] Bau, O. and Mackay, W. E. 2008. OctoPocus: A dynamic guide for learning gesture-based command sets. In Proc. UIST 2008. ACM, New York, NY, 37-46.

[2] Brown, Q., Bonsignore, E., Hatley, L., Druin, A., Walsh, G., Foss, E., Brewer, R., Hammer, J., and Golub, E. 2010. Clear Panels: a technique to design mobile application interactivity. In Proc. DIS 2010. ACM, New York, NY, 360-363.

[3] Cao, X. and Zhai, S. 2007. Modeling human performance of pen stroke gestures. In Proc. CHI 2007. ACM, New York, NY, 1495-1504.

[4] Chang, C.-K. 2008. Usability comparison of pen-based input for young children on mobile devices. In Proc. SUTC 2008. IEEE, New York, NY, 531-536

[5] Kellogg, R. 1970. Analyzing Children's Art. Mayfield Pub Co., CA, USA.

[6] Long, A. C., Landay, J. A., Rowe, L. A. and Michiels, J. 2000. Visual similarity of pen gestures. In Proc. CHI 2000. ACM, New York, NY, 360-367.

[7] Lowenfeld, V. 1987. Creative and Mental Growth. Prentice Hall, NJ, USA.

[8] McKnight, L. and Cassidy, B. 2010. Children's interaction with mobile touch-screen devices: experiences and guidelines for design. International Journal of Mobile HCI 2 , 2 (2010), 1-18.

[9] McKnight, L. and Fitton, D. 2010. Touch-screen technology for children: Giving the right instructions and getting the right responses. In Proc. IDC 2010. ACM, New York, NY, 238-241.

[10] Read, J. C. 2007. Children using digital ink for writing. In Proc. PLT 2007. IEEE, New York, NY, 1-5.

[11] Ren, X. and Zhou, X. 2011. An investigation of the usability of the stylus pen for various age groups on personal digital assistants. Behaviour \& Information Technology 30, 6 (2011), 709-726.

[12] Revelle, G. and Reardon, E. 2009. Designing and testing mobile interfaces for children. In Proc. IDC 2009. ACM, New York, NY, 329-332.

[13] Shelly, G. B. and Vermaat, M. E. 2010. Discovering Computers 2010: Living in a Digital World, Fundamentals. Cengage Learning, MA, USA, 2010.

[14] Tu, H., Ren, X., and Zhai, S. 2012. A comparative evaluation of finger and pen stroke gestures. In Proc. CHI 2012. ACM, New York, NY, 1287-1296.

[15] Wobbrock, J. O., Wilson, A. D., and Li, Y. 2007. Gestures without libraries, toolkits or training: A $\$ 1$ recognizer for user interface prototypes. In Proc. UIST 2007. ACM, New York, NY, 159-168. 